\title{
Building international partnerships
}

\author{
X. X. Zheng' MD, A. S. Hinshaw ${ }^{2}$ PhD, RN, FAAN, M. Y. Yu ${ }^{3}$ PhD, MD, \\ G. F. Guo ${ }^{4}$ MD \& D. J. Oakley ${ }^{5} \mathrm{PhD}$ \\ I Dean, Peking University School of Nursing, Beijing, China \\ 2 Dean, University of Michigan School of Nursing, Ann Arbor, Michigan, USA \\ 3 Research Faculty, University of Michigan School of Nursing, Ann Arbor, Michigan, USA \\ 4 Visiting Scholar and Faculty, Peking University School of Nursing, Beijing, China \\ 5 Professor, University of Michigan School of Nursing, Ann Arbor, Michigan, USA
}

Keywords

Community,

International

Partnerships, Nursing

Education
Correspondence address:

Dr Mei-yu Yu, Grants and Research Office, University of Michigan School of Nursing, 400 N. Ingalls, Ann Arbor, MI 48109-0482, USA Tel.: (734) 936-8913 Fax: (734) 615-1666 E-mail: yujiang@umich.edu

\section{Abstract}

Health is a global concern. Although nursing is a global profession, most schools of nursing concentrate on teaching health exclusively within the context of their own nation. Sister-school partnerships that cross national boundaries are one way of extending the learning opportunities of faculties and students. An example of a 5 -year partnership is described and analysed.

\section{Introduction}

International partnerships provide many opportunities for the schools, faculties and students involved. The usual objectives are to expand faculty and student learning, to stimulate collaborative multisite research studies and to benefit the curricular offerings, student recruitment, or funding base of the home institution. At present, health problems extend beyond national borders, and nurses need to provide health care for diverse populations (Orchard \& Karmaliani 1999).

International work is complex. Any crossnational collaboration requires flexibility and sophistication (Basch 1999). International work also requires an understanding of cultural differences, including language, lifestyle, assumptions about health, economic resources and political systems (Traynor \& Rafferty 1999). It also requires additional resources of time and, often, of money (Basch 1999).

\section{The history of one partnership}

As part of a national change in policy (Lin 1998), Beijing Medical University re-established a Depart- ment of Nursing in 1984, with baccalaureate students enrolled in 1985. The Department of Nursing was the first in China to offer a Master's Degree in Nursing, starting in 1990. In 1999, the Department was awarded the status of School of Nursing. In 2000, Beijing Medical University and Beijing University merged and the former name, Peking University, was restored. For the academic year 1999-2000, 1100 students were enrolled in one of the following four programmes offered by the Peking University School of Nursing: Master of Science in Nursing, Baccalaureate of Science in Nursing, Associate/Vocational Practice Nurse Training and the Programme from the Associate to Baccalaureate. The School has 67 faculty members, of whom nine have senior professional titles and seven have achieved Masters of Science in Nursing. The faculty are leaders in publishing academic papers in the 13 nursing professional journals (Chang 1999) in China. The Peking University School of Nursing is also the author of textbooks, instructional manuals and reference books on topics such as nursing diagnosis, nursing administration, nursing research methodology, etc. These are now widely used in the China Television University, in national self-teaching programmes 
and by nursing students in other schools of nursing.

The University of Michigan School of Nursing, established in 1891, became an independent school of nursing within the university in 1941. For the academic year 1999-2000, 865 students were enrolled, of whom two-thirds were undergraduates. In addition to over 200 master's students, there are almost 80 doctoral students and six postdoctoral fellows. There are 114 faculty members, many of whom are well known for their publications in health promotion, doctoral education and nursing research. The school has recently been ranked among the top five schools of nursing in the USA.

Leaders of both universities have been striving to expand the global emphasis of their students' educational experiences. Whilst the global economy is an important basis for this leadership goal, exchange of scientific information is fast becoming an important resource for improving the quality and effectiveness of health care. In China, government and academic leaders encourage international partnerships. In the USA, the population requiring nursing services is increasingly diverse. As the population of Chinese descent is increasing rapidly in the service area of the University of Michigan, the University of Michigan School of Nursing had a particular interest in building a close relationship with a school of nursing in China.

Individual faculty visits, starting as early as 1987 , culminated in an official partnership between the Faculty of Nursing of Beijing Medical University (now Peking University School of Nursing) and the School of Nursing of the University of Michigan. In 1996, the two Deans signed a formal agreement that had the approval of the key academic administrators of their universities. The objectives were to:

- provide academic learning for faculty members through workshops, lectures and consultation through short-term programmes,

- improve the education of undergraduate and graduate students in both institutions,

- co-operate in cross-cultural nursing research and application of that research to nursing practice, and - address other matters of mutual agreement.

The two schools co-operated in writing grant proposals to a private foundation that had a small programme for funding health initiatives in China. With relatively limited funding, the partnership built on a number of programmes and common research interests. The theme of this collaborative work was community-based nursing care because that is a priority in China's health system, a particularly strong specialty at the University of Michigan and a priority for the funding agency.

The partnership started with a joint workshop for the nursing faculty and administrators from the Beijing area. The sessions included overviews of nursing education and research in the USA. In addition, audience members participated in planning a nursing research agenda that was relevant for nurses in the Beijing area. A second collaborative workshop, on women's health, was held in Beijing in 1996; a third was provided on the nursing care of dementia patients in 1998; and a fourth was conducted collaboratively, on community-based nursing care, in 1999. The 1998 workshop on dementia and the 1999 workshop on communitybased nursing care were provided for national audiences, with representatives from 16 provinces. Presentations were always collaborative.

The Peking University School of Nursing was provided with administrative reimbursement for each workshop, which was used to cover the cost of all correspondence, registration processing, workshop materials, faculty translators and the evaluation report. The project also reimbursed the travel expenses of the speakers from the University of Michigan School of Nursing.

A key to the partnership has been the exchange visits by the two Deans. The first invitation was made in 1996, by the then Beijing Medical University to the University of Michigan. In 1997, the Dean and the Associate Dean of Beijing Medical University visited the University of Michigan. Through these visits, the leading administrators in each university learned about the programmes in the partner schools. The Deans also established the substantive basis for continuing to work together. Through seminars provided by each Dean at the partner school, the faculty of each school also learned about the status of nursing and nursing education in the other nation. 
Another major component of the partnership has been a programme of visiting scholars, whereby Peking University School of Nursing selects a nurse faculty member for a one-semester learning experience at the University of Michigan. By spring 2000, a total of five nurses from the Peking University School of Nursing faculty had spent 4 months each at the University of Michigan. The programme undertaken by the visiting scholar always includes classroom observation, as designated by the Dean of the Peking University School of Nursing. That is, during the month or two before the scholar's trip, all classes (undergraduate as well as graduate) offered by the University of Michigan in the relevant semester are e-mailed to the Dean of the Peking University School of Nursing. Based on that school's needs and the visiting scholar's area of expertise, appropriate courses are selected. It is the job of the University of Michigan faculty project leaders to contact the course instructors to ensure that an additional person can join the classroom or clinical experience. The sister-school partnership has been immensely enriched by the willingness of the individual faculties to accept the scholars. In turn, scholars usually contribute to the class through a special lecture or informal sharing about health conditions and nursing in China. All visiting scholars also present a seminar on some aspect of nursing care or education in China.

Travel and living expenses for each visiting scholar are provided, as is a small stipend for each month in residence. Travel and housing arrangements are made by the University of Michigan's project staff.

The visiting scholars also participate in a research project with the University of Michigan faculty of Nursing. Several have co-authored papers. Some staff of the faculty of Nursing at the University of Michigan have conducted research projects in China and among Chinese-Americans, and the cultural and language knowledge of the Peking University School of Nursing scholars has added substantively to this research. This type of research collaboration uses and extends the research knowledge of faculty members about matters such as new technologies for data collection, entry, analysis and manuscript preparation.
In addition, the visiting scholars each observe innovative nursing care in community settings. These include nurse-managed clinics associated with the University of Michigan, hospice services, long-term care facilities and state-wide demonstration projects to promote primary care. The visiting scholars have also helped to improve communication between the local Chinese-American communities and the University by interacting informally with the increasing numbers of Chinese nurse graduates now working in the Ann Arbor area. As a result of this, the scholars are well positioned to identify biculturally competent nurses who could become resources for teaching in China in the future.

When the visiting scholars return home, they take with them boxes of current books to expand the nursing library at the Peking University School of Nursing. Course syllabi and readings are also part of the partnership. As the partnership's emphasis is on community-based nursing, there has been useful consultation about additional undergraduate curricular material required by the Peking University School of Nursing on community health, and there has been consultation about assessing community health care needs in a nearby neighbourhood. A survey conducted by the Peking University School of Nursing documents needs that have not yet been met by existing health services. The results provided direction and justification for an innovative proposal to establish a nurse-managed, communitybased clinic as a teaching site in Beijing.

A distinctive aspect of the partnership has been the development of collaborative research. The first project was pilot research on family planning, proposed by a University of Michigan faculty member and conducted by the Peking University School of Nursing faculty. The results were incorporated into a National Institutes of Health (NIH) grant proposal, but as it was not funded, the project was not continued. A second project involved data that had been collected in China by the University of Michigan faculty, with the visiting scholars involved in data analysis and manuscript preparation (Oakley et al. 1999).

The third project is the first to be truly collaborative. The workshop on dementia care led to the joint development of a project plan that replicates, in 
China, part of an NIH-funded US research project among dementia patients, with protocols based on established US procedures and protocols. The study is designed to determine whether US and Chinese specialists, who are involved in the nursing care of the elderly, classify dementia behaviours in the same way. A standardized coding scheme has been developed. Training for this study uses translated/ back-translated assessment tools, bilingual written protocols for coding videotaped behaviours and cross-national evaluations of pilot cases. This project is supported by a small grant from a private healthcare foundation. Limited travel, equipment and research costs are provided for both institutions.

In addition to these educational and research activities, the collaboration has facilitated a yearlong sabbatical for a professor from the Foreign Language Department of Peking University, who teaches English to nursing students there. Funded by the Chinese Government, she spent a year at the University of Michigan's School of Nursing to develop a Chinese-English dictionary of health care terms. In this effort, she worked with several faculty specialists of the University of Michigan to ensure that the health care terms were translated appropriately to convey the deeper meanings.

\section{Evaluating the partnership}

Visiting scholars complete an Expectations Form upon arrival at University of Michigan and an Outcomes Evaluation Form shortly before departure. As our experience grows, the learning experiences are rated as more applicable to the needs of nursing within China. Also, small changes in living arrangements and daily activities have resulted from the evaluations.

The partnership is evaluated by the Deans each time they meet and in preparation for biennial grant renewal applications. The partnership is considered a success because it has made contributions to the educational programmes at both institutions. In addition to the enriched experience of those direct participants at both schools, co-authored publications and collegial research projects have begun. In addition, each school has expanded its collabora- tions to include other partners and funding sources. These developments indicate that each institution has helped the other to gain independent strength for collaborative work on behalf of nursing.

These gains have required costs beyond the financial support provided by external funding. Each side has devoted faculty time and facilities to the partnership. The faculty time has far exceeded the amount reimbursed from our small grant. However, the faculties of both schools increasingly believe that their curricular enrichment and individual learning opportunities make the partnership worthwhile. In addition to the expanded hours devoted to community health nursing in the undergraduate curriculum at the Peking University School of Nursing, numerous students have used data from the neighbourhood survey for their student theses. In their formal evaluations, all of the visiting scholars noted specifically that they had obtained information/materials that could enhance nursing education in China, acquired new teaching methods and learned about nursing curriculum issues. Ongoing subscriptions to key nursing journals and teaching videos have been part of the project. The programme has also facilitated the transmission of hard-to-find scientific literature listed in database searches. At the University of Michigan, the talks made by visiting scholars led one faculty member to link her undergraduate RN students with Peking University School of Nursing students to learn first-hand about the health care system in China. Via e-mail, students exchange questions and answers, and knowledgeable faculty members are available to help ensure an understanding of the contexts. A doctoral student at the University of Michigan was inspired by a visiting scholar to try to arrange a short-term internship experience in Beijing, a plan that is still in process. The relationship has also led to further diverse projects, including other sister-school relationships for both schools and industry-sponsored research.

The project has laid the groundwork for a new, model, community-based clinic at Peking University. The clinic is a new phase of our partnership. Location, services and staffing are being negotiated by Peking University's School of Nursing. Consultation is provided from the University of Michigan's 
experience in building a state-wide consortium of eight academic nurse-managed centres. Financial support has been received from the W. K. Kellogg Foundation. The sister-school partnership has also facilitated the development of successful demonstration and research projects with ChineseAmericans in the state of Michigan. The State of Michigan provides support for a demonstration and research programme to expand the use of mammography for breast cancer screening of AsianAmerican women. The US government's National Cancer Institute provides support for a research programme testing a community-based approach to recruiting Chinese-American women for breast and cervical cancer prevention programmes. These two complementary programmes are part of the Healthy Asian-Americans Project (HAAP). Visiting scholars participated in the early development of HAAP and, in turn, some local nurses of Asian ancestry who are participating in HAAP have been drawn into hosting and other activities with the visiting scholars. Because of the partnership, each school has gained stature within its own university. The collaborative activities are seen as meaningful and important to each university as a whole. Leaders of the project have been designated as Visiting or Adjunct Professors of the partner University; and project staff at the University of Michigan have, for the first time, been named as Associates of the University of Michigan's Center for Chinese Studies.

\section{Conclusion}

Although each nation has its own political, economic and cultural setting for nursing education, the principles of nursing are universal (Traynor \& Rafferty 1999). Global trade and immigration make it essential for nurses to know about innovations and issues in nursing in diverse settings. Both schools have obtained specific benefits.

Although there are multiple ways to learn about other cultures, partnership links between schools of nursing are essential for true professional collaboration. We have learned that partnerships require work, money, flexibility and understanding. Our joint practice has been to have leadership meetings once every 2 to 3 years. Electronic communication and communication through consultations with faculty and project staff are sufficient to make the project operate day-by-day and month-by-month. However, strategic decision making must be well prepared at the staff level so that clear conclusions can be agreed upon during official meetings of the Deans.

An important aspect of our project has been mutual respect, as well as common interests and goals. An important lesson has been that each step has required more time than planned. Decisions have been made that required great patience and understanding. Hence, bicultural learning and acceptance is an important requirement.

\section{Acknowledgements}

Funds for partnership activities have been provided by the W. K. Kellogg Foundation and Blue Cross/ Blue Shield of Michigan.

\section{References}

Basch, P.F. (1999) Textbook of International Health. Oxford University Press, New York.

Chang, W.Y. (1999) Interview of Visiting Health Administrators from the Ministry of Health, Beijing, China. Image: Journal of Nursing Scholarship, 31, 185-187.

Lin, J.Y. (1998) A Future Perspective of the Development of Nursing in China Towards the Twenty-First Century. Hong Kong Nursing Journal, 34, 35-37 (in Chinese).

Oakley, D., et al. (1999) Combining Qualitative with Quantitative Approaches to Study Contraceptive Pill Use. Journal of Women's Health, 8, 249-257.

Orchard, C.A. \& Karmaliani, R. (1999) Community Development Specialists in Nursing for Developing Countries. Image: Journal of Nursing Scholarship, 31, 295-299.

Traynor, M. \& Rafferty, A.M. (1999) Nurse Education in an International Context: the Contribution of Contingency. International Journal of Nursing Studies, 36, 85-91. 